\title{
Second derivative multistep method for solving first-order ordinary differential equations
}

\begin{abstract}
In this paper, a new second derivative multistep method was constructed to solve first order ordinary differential equations (ODEs). In particular, we used the new method as a corrector method and 5-steps Adamôs Bashforth method as a predictor method to solve first order (ODEs). Numerical results were compared with the existing methods which clearly showed the efficiency of the new method.
\end{abstract}

Keyword: Second derivative multistep method; First order ordinary differential equations 